Instituto de Investigación en Ciencias Odontológicas, Área de Ciencias del Comportamiento, Facultad de Odontología, Universidad de Chile. aMA, Psicólogo. bMsC, Médico Cirujano. 'MsC, Cirujano Dentista, Psicólogo.

Recibido el 26 de septiembre de 2013, aceptado el 27 de agosto de 2014.

Correspondencia a: Andrea Herrera Facultad de Odontología, Universidad de Chile. Sergio Livingstone 943 , Independencia, Santiago. Celular: 90894050 Teléfono-Fax: 29781844 aherrerar@u.uchile.cl

\section{Entrega de malas noticias en la práctica clínica}

\author{
ANDREA HERRERAa, MATÍAS RÍOS ${ }^{a}$, \\ JOSÉ MANUEL MANRÍQUEZ ${ }^{\mathrm{b}}$, GONZALO ROJAS
}

\section{Breaking bad news in clinical practice}

Breaking bad news is a complex task that requires multiple communication skills from health professionals. Clinical practice demands to communicate all type of bad news, from a diagnosis of cancer to adverse effects of a treatment. On the other hand, since the beginning of the health reform in 2003, the need to improve the quality of services was proposed, among which the concern about the rights and duties of patients stands out. Therefore, the health care providerpatient relationship becomes again the subject of discussion and study, and a topic of great importance for clinical work. We revise the consequences of breaking bad news for the patient and for the health care provider, as well as the current protocols available for this purpose. The importance of developing communication skills both for future health professionals as for those who currently work in the area is emphasized.

(Rev Med Chile 2014; 142: 1306-1315)

Key words: Health communication; Interpersonal relations; Truth disclosure.
I a comunicación de malas noticias es un aspecto central de la práctica clínica. Los profesionales de la salud deben rutinariamente comunicar malas noticias, como resultados anormales de exámenes, malos pronósticos, resultados adversos de los tratamientos, entre otros ${ }^{1}$. A pesar de lo habitual de esta práctica, es considerada una tarea complicada ${ }^{2}$ y poco confortable ${ }^{1}$.

La entrega de malas noticias es una tarea de comunicación compleja que requiere de otras habilidades además de las verbales ${ }^{3-4}$, como es el responder a la reacción emocional del paciente, involucrarlo en la toma de decisiones, manejar el estrés generado por las expectativas de cura del paciente, involucrar a los miembros de la familia en el proceso de salud-enfermedad, y el dilema de cómo dar esperanzas cuando la situación es poco prometedora ${ }^{3}$.

El incremento de las enfermedades crónicas y de los temas relacionados con la calidad de vida, sumado a la comprensión de la enfermedad desde un enfoque biopsicosocial, han aumentado la importancia de entender cómo la entrega de malas noticias afecta a los pacientes, a su familia/cuidadores y a los profesionales de la salud ${ }^{5}$.

\section{Entrega de malas noticias}

La mala noticia puede tener diferentes significados para diferentes personas, como por ejemplo a un paciente al que se le dice que es VIH positivo, un hombre al que se le dice que su padre tiene Alzheimer, a un paciente que se le diagnostica un cáncer de pulmón, a una pareja que se le dice que no pueden tener hijos, entre otros ${ }^{4}$. Las malas noticias pueden incluir la entrega de información respecto a la recurrencia de una enfermedad, propagación de una enfermedad, o la falla del tratamiento que impide la proliferación de la enfermedad, la presencia de efectos colaterales irreversibles, o resultados de test genéticos adversos ${ }^{4}$.

Existen numerosas definiciones de malas noticias. Días 6 la define como "cualquier información que afecta adversa y seriamente la visión del individuo de su futuro", mientras que Bor, Miller, 
Goldman y Scher" la plantean como una "situación donde hay un sentimiento de no esperanza, una amenaza al bienestar mental o físico de la persona, riesgo de alterar un estilo de vida establecido o donde el mensaje entregado transmite pocas opciones de vida" (p. 70).

El común denominador es que el mensaje es una mala noticia, la cual tiene el potencial de destruir esperanzas y sueños, y que conducen a estilos de vida y futuros muy diferentes a los que se tienen ${ }^{4}$. El profesional de salud no puede estimar el impacto que tendrá la mala noticia hasta que no determina primero las expectativas de recibirla o el entendimiento de la situación por parte del paciente.

Es importante aceptar que la mala noticia no se puede convertir en buena noticia ${ }^{8}$. El grado de la mala noticia es proporcional a la distancia entre la percepción del paciente de la situación y la realidad ${ }^{9}$ por lo que la tarea del profesional de la salud es facilitar el paso de la percepción de su situación hacia la realidad ${ }^{8}$.

\section{Consecuencias de una mala noticia}

La secuela de una mala noticia entregada de manera abrupta e intensa puede ser devastadora y de larga duración ${ }^{10}$. Los estudios consistentemente han demostrado que el modo en que el profesional de la salud entrega la mala noticia, establece una marca indeleble sobre la relación profesionalpaciente 4 . Cuando la noticia es entregada pobremente, la experiencia puede quedar en la mente del paciente y/o su familia por largo tiempo ${ }^{11}$.

El cómo la notica es discutida o conversada con el paciente puede afectar la comprensión de la información ${ }^{3,12-15}$, del pronóstico de la enfermedad $^{14-15}$, la satisfacción con la atención médica ${ }^{15-16}$, el nivel de esperanza frente a la enfermedad ${ }^{17}$, y el posterior ajuste o adaptación psicológica ${ }^{18-23}$.

Las habilidades comunicacionales no efectivas entre el profesional de la salud y el paciente pueden llevar a estrés o incluso a un aumento de la ansiedad y depresión en el paciente. Algunas formas específicas de consolar, dar tranquilidad o seguridad, así como la inadecuada entrega de información, han demostrado un incremento en la ansiedad de los pacientes inmediatamente después de la consulta médica ${ }^{21,24-26}$.

Por otra parte, el portador de la mala noticia experimenta fuertes emociones como ansiedad, responsabilidad por la noticia y miedo a la evaluación negativa. Es decir, entregar malas noticias puede ser sumamente estresante para el profesional de la salud involucrado. Estudios muestran que una de las mayores causas de estrés en el equipo de oncología es la comunicación con pacientes y familiares ${ }^{27-28}$. El estrés generado crea una resistencia a entregar este tipo de noticias, que puede llevar al profesional a evitar discutir información estresante o evitar temas sensibles como un diagnóstico desfavorable, el pronóstico, o, transmitir optimismo injustificado, lo que contribuye al falso sentido de esperanza del paciente $e^{3,29}$.

La esperanza es importante para los pacientes, no obstante, los médicos aún están algo inseguros del cómo promover este factor frente a una enfermedad que amenaza la vida. La esperanza en medicina es de dos tipos: específica (por un resultado específico) y generalizada (un sentido de optimismo inespecífico). Al momento del diagnóstico de una enfermedad terminal, el médico puede mantener un optimismo general, el cual es compatible con la obligación del médico de decir la verdad. Este optimismo puede ayudar sustancialmente al paciente y su familia a la adaptación con la realidad. El médico puede ayudar al paciente y su familia estableciendo objetivos específicos como mejorar el medioambiente del paciente en el hospital, el control del dolor y el alivio del insomnio ${ }^{30}$. Los médicos pueden y deben promover el optimismo sin avalar una esperanza poco realista ${ }^{30}$.

Entregar malas noticias puede ser aún más estresante cuando se es inexperto o se tiene poca experiencia ${ }^{6}$, con pacientes jóvenes, cuando hay una relación de larga data con el paciente ${ }^{6}$, cuando previamente se ha expresado un fuerte optimismo del éxito de los resultados, y cuando las perspectivas de un tratamiento efectivo son limitadas ${ }^{3,6}$. Se ha indicado que aquellos que tienen dificultades en la entrega de malas noticias, pueden ser propensos a ofrecer y prestar un trato duro ${ }^{3}$.

La percepción del profesional de la salud de cuáles son los objetivos importantes para el paciente también influencian el cómo se entrega la noticia ${ }^{1}$. Una pobre comunicación puede frustrar el objetivo de entender las expectativas de tratamiento del paciente o involucrar al mismo en la planificación del tratamiento.

La evidencia sugiere que los profesionales de 
la salud se incomodan con la discusión de malas noticias debido a las siguientes razones:

- Incertidumbre acerca de las expectativas del paciente

- Miedo a destruir el optimismo del paciente

- Miedo a una reacción inadecuada

- Vergüenza por haber entregado previamente una visión demasiado optimista al paciente

- Miedo a su propia ineficiencia en términos de la incontrolabilidad de la enfermedad ${ }^{3}$

- Sentirse no preparado para manejar la reacción emocional anticipada del paciente ${ }^{3}$

Según la perspectiva de los pacientes, los factores que contribuyen a una entrega pobre de una mala noticia son: la excesiva franqueza, lugar y/o tiempo que no conduce a tener una discusión seria, y la falta de mantenimiento del optimismo. Enfatizan la importancia de moverse entre la honestidad y el realismo con sensibilidad y apoyo ${ }^{31}$.

\section{Habilidades comunicacionales}

Las habilidades comunicacionales en la entrega de la información son requeridas mayormente cuando hay una divergencia entre la perspectiva del profesional y su paciente. La entrega de malas noticias es un ejemplo de esa situación: El paciente espera entrar a la consulta focalizado en la posibilidad de recibir buenas noticias, y el profesional tiene que gradualmente movilizar la atención del paciente hacia los hechos preocupantes que él ahora debe comenzar a comunicar ${ }^{10}$.

La comunicación abierta entre el profesional de la salud y su paciente es crítica para desarrollar una relación terapéutica, obtener información e implementar un plan de tratamiento. Este nivel de comunicación requiere confianza mutua y respeto, al igual que importantes habilidades de escucha ${ }^{29}$.

Dos factores importantes en la entrega de malas noticas son el deseo de discutir sobre la muerte y la sensibilidad hacia la dificultad del tema. Se debe no sólo entregar la información claramente, sino que también, entregar apoyo emocional, responder a las reacciones del paciente y su familia, calmando, aliviando, disminuyendo cualquier temor de abandono por parte del profesional, participar en la toma de decisión grupal y mantener el optimismo ${ }^{29}$.

El éxito de la entrega o discusión de la noticia se realiza en base a los objetivos, los cuales pueden variar entre paciente y profesional de la salud. Sin embargo, en la literatura hay poca mención explícita en relación a los objetivos y cuando se hace, se mencionan indirectamente a través de las medidas de resultados usados para definir la comunicación como exitosa ${ }^{1}$.

Sweeny, Shepperd \& $\mathrm{Han}^{1}$ evaluaron seis potenciales objetivos en la entrega de una mala noticia, en base a la revisión de la literatura, y exploraron la percepción tanto de pacientes como de médicos de cuan importantes son en la comunicación. Estos objetivos son:

- Entregar información: los pacientes necesitan información clara y exacta para tomar decisiones informadas y planificar el futuro ${ }^{32-33}$.

- Persuadir al paciente de adoptar las recomendaciones: en muchas circunstancias clínicas, hay un curso óptimo de acción asociado con un balance favorable de beneficios vs daños. En estas circunstancias los profesionales de la salud, pueden desear (consciente o inconscientemente) persuadir al paciente hacia estas acciones, y cada persuasión puede llegar a ser parte de la discusión de la mala noticia. Los profesionales deben ser conscientes de sus intenciones de persuasión y explicitarlas al paciente ${ }^{31,34,35}$.

- Minimizar el estrés del paciente: escuchar malas noticas puede ser abrumador emocionalmente para el paciente, y los profesionales pueden exacerbar este estrés si entregan de mala manera dicha noticia ${ }^{36}$.

- Promover la satisfacción del paciente: la satisfacción del paciente es un indicador de la calidad de la comunicación profesionalpaciente ${ }^{37-39}$. Los factores que pueden influenciar la satisfacción son la discusión, proveer responsividad a las emociones del paciente e involucrar al mismo en la toma de decisiones.

- Mantener el optimismo del paciente: promover el optimismo del paciente ${ }^{35,40}$, el cual puede ser un poderoso predictor de adaptación y recuperación ${ }^{41}$ aunque debe ser balanceado con honestidad y realismo ${ }^{39,40}$.

- Minimizar el discomfort del profesional de la salud: el objetivo final del profesional de la salud debe ser minimizar su propio discomfort con la tarea, el cual puede resultar en miedo a la reacción al paciente, a sus propias emociones, a su falta de competencia o miedo a ser cul- 
pado $^{42}$. Reducir este discomfort puede influir sobre el bienestar emocional, satisfacción y competencia del profesional de la salud con la entrega de malas noticias ${ }^{43}$.

La tarea de la entrega de malas noticias puede ser mejorada a través de entender el proceso que involucra el enfoque de protocolos de procedimiento, aplicando principios bien establecidos de comunicación y consejería ${ }^{3}$.

Existe mucha literatura en inglés sobre estrategias de cómo dar malas noticias ${ }^{3,44-47}$ sin que exista consenso acerca de cuál es la más adecuada, lo cual refleja la multiplicidad de posibles objetivos implícitos en esta tarea.

\section{Protocolos}

A continuación se presentan dos protocolos de entrega de malas noticias.

\section{SPIKES:}

Fue desarrollado por Baile et al. ${ }^{48}$, basado en el protocolo de Bukman ${ }^{10}$. SPIKES es una sigla en inglés, cada letra representa una fase en este esquema de 6 pasos: 1) S setting; 2) P perception; 3) I invitation; 4) K knowledge; 5) E empathy y 6) S summary (ver Tabla 1).

Fue desarrollado para presentar información estresante de una manera organizada a los pacientes con cáncer y su familia. Entrega un orden paso a paso para la discusión difícil, como lo es la recurrencia del cáncer, o cuando las opciones de tratamiento se han agotado y el cuidado paliativo es el indicado ${ }^{3,49,50}$.

Los componentes esenciales de SPIKES incluyen demostrar empatía, reconocer y validar los sentimientos del paciente, explorar el entendimiento y aceptación de la mala noticia, y entregar información sobre posibles intervenciones ${ }^{51}$.

Los objetivos del protocolo son, en primer lugar, obtener información del paciente, lo que permitirá determinar el conocimiento, las expectativas del paciente y su disposición a escuchar malas noticias. El segundo objetivo es entregar información legible acorde a las necesidades del paciente y sus deseos. El tercer objetivo es apoyar al paciente en el empleo de habilidades para reducir el impacto emocional y el aislamiento experimentado por recibir la mala noticia. El objetivo final es desarrollar una estrategia en la forma de un plan de tratamiento con la cooperación del paciente ${ }^{3}$.

No todos los episodios de entrega de malas noticias requieren de todos los pasos del protocolo, pero cuando se hacen se debe seguir la secuencia ${ }^{3}$.

Este protocolo ha sido aplicado en distintos países como por ejemplo Alemania ${ }^{52}$, Francia ${ }^{53}$, China $^{54}$, Estados Unidos de Norteamérica ${ }^{51}$, entre otros, y en distintos contextos además del cáncer, como lo son la psiquiatría ${ }^{55}$, oftalmología ${ }^{56}$, veterinaria ${ }^{57}$ y la odontología ${ }^{58}$. En la Tabla 1 se muestra un compendio con lo expuesto por los autores Kaplan $^{51}$, Baile et al. ${ }^{3}$ y DHSSPS ${ }^{4}$ sobre SPIKES.

\section{Bennett \& Alison ${ }^{8}$}

Los autores sugieren un enfoque de 7 pasos para discutir el diagnóstico de cáncer con el paciente basado en su propia experiencia y la recomendación de otros. Los pasos pueden ser adaptados a una variedad de situaciones clínicas donde la mala noticia es entregada y no está diseñado solamente para la discusión del diagnóstico de cáncer (Tabla 1).

Además hacen recomendaciones con respecto a la negación y la colusión con parientes. La negación puede ser un mecanismo de afrontamiento útil para el paciente que no puede adaptarse a la información dada. Llega a ser un problema cuando causa angustia significativa al paciente o familiares, o si impide al paciente manejarse con temas importantes para él y su familia. Una exploración o indagación sensible y asertiva de la historia, y confrontarlo con las incongruencias entre sus síntomas y su explicación, puede ser suficiente para quebrar la negación de una adecuada manera.

La colusión con parientes es una conspiración de silencio que puede contribuir a la soledad y a una muerte aislada, si al paciente no se le da la oportunidad de saber su diagnóstico. La presión de los parientes de mantener el secreto puede ser inmensa, por lo que es importante desafiar la colusión para ahorrarle al paciente la ansiedad y la angustia del aislamiento y evitar un duelo complicado para los parientes, quienes fueron prevenidos o avisados de ser abiertos con el paciente antes de la conspiración. Éticamente la información clínica debe ser discutida con el paciente primero, y únicamente puede ser discutida con la familia si el paciente da la autorización para hacerlo. 


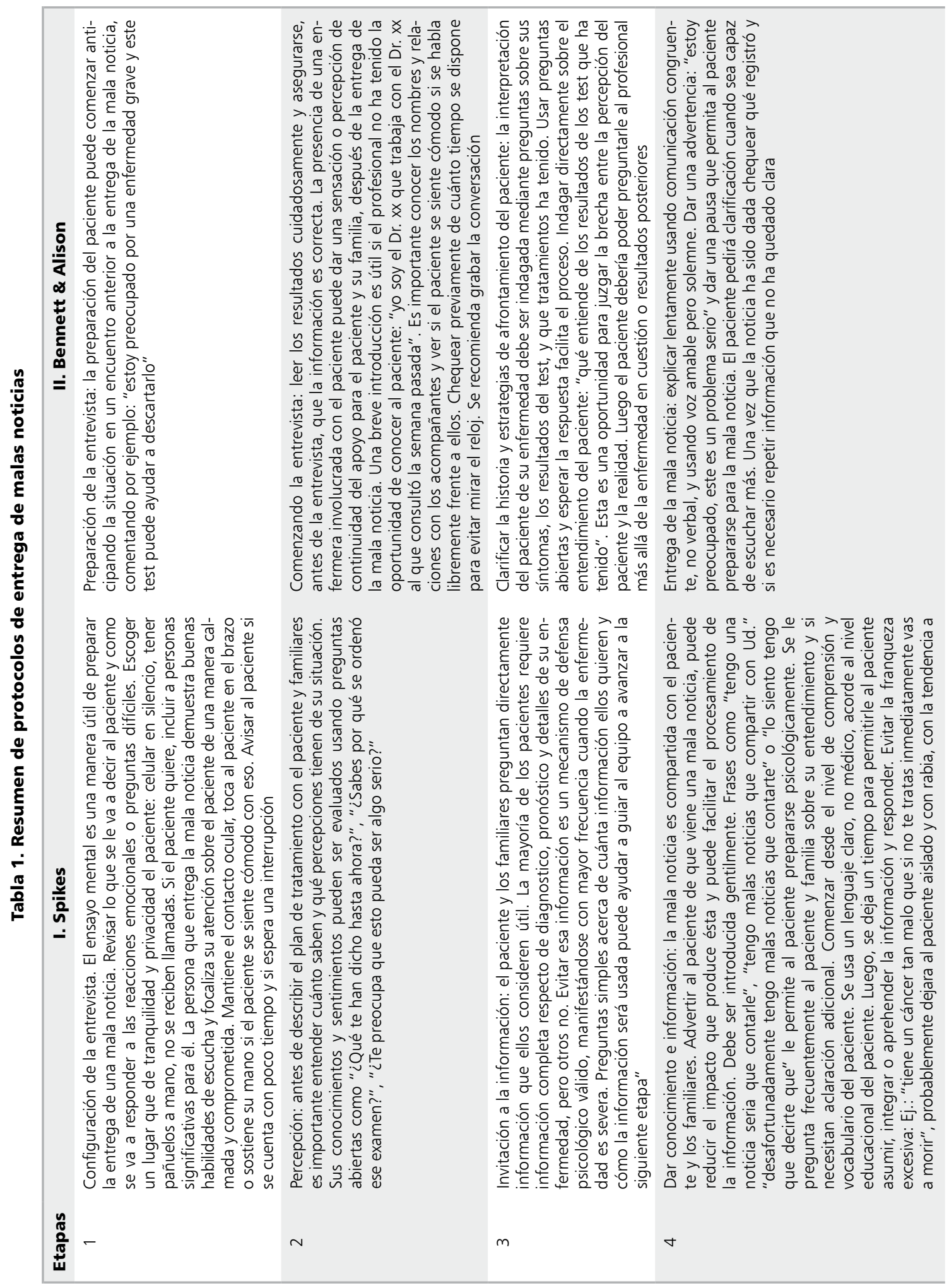




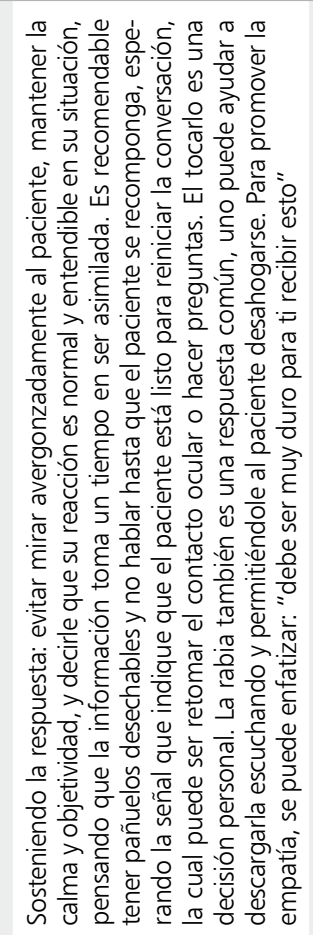

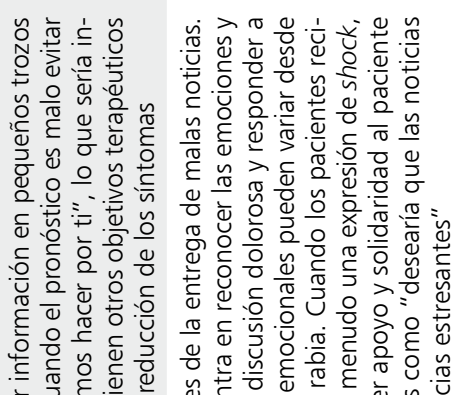

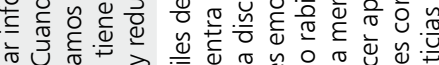

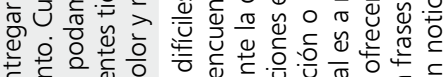

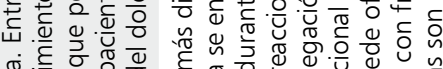

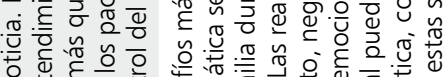

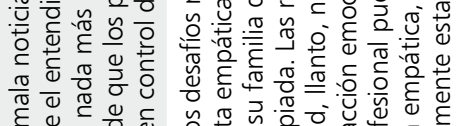

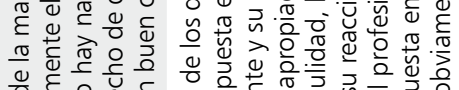

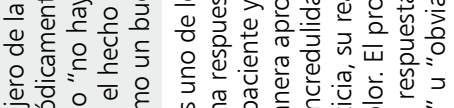

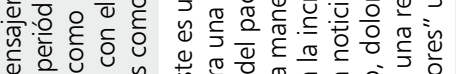

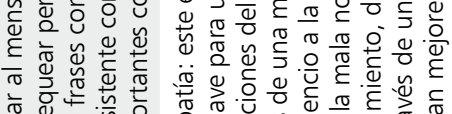

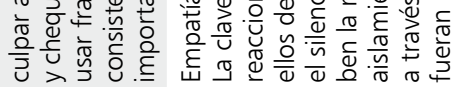

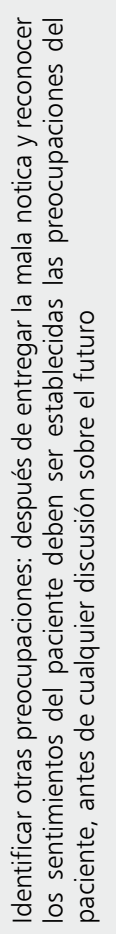

dั 응 岕芯

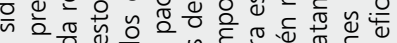

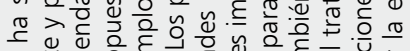

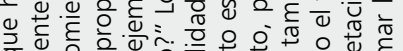
大

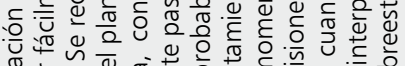
हैं

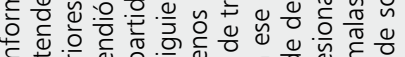

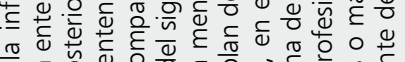

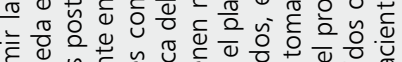

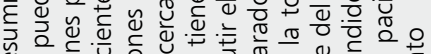

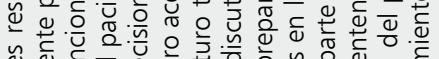

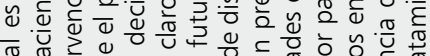

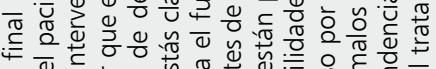

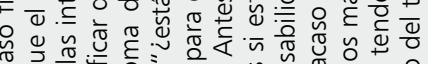

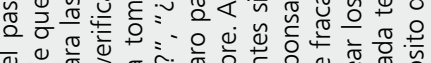

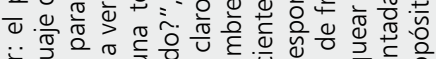

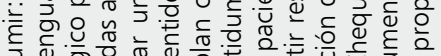

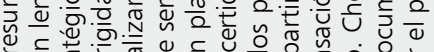
$>$ >

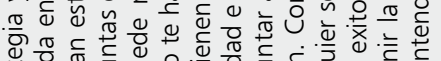

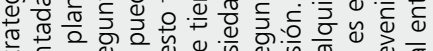

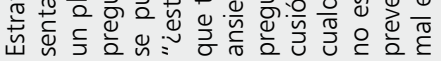

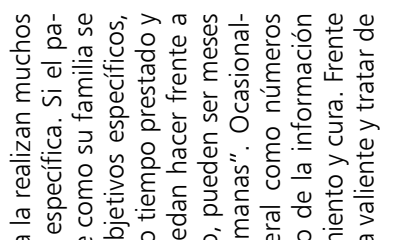



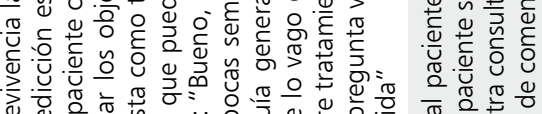

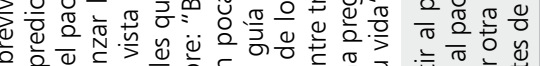

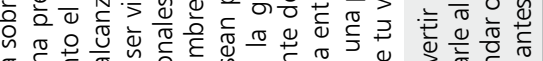

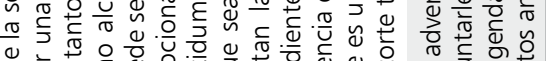

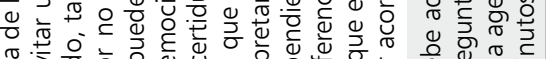

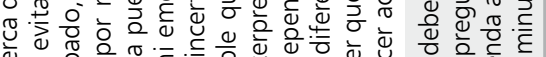
嵌

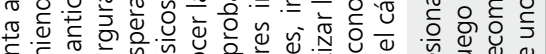

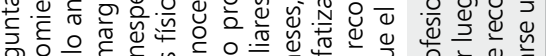

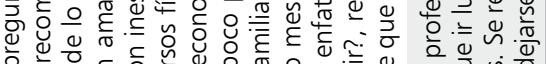
은 ○े 琎宁

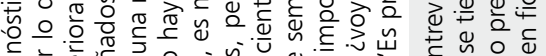

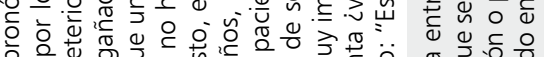

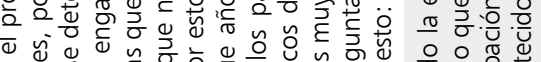

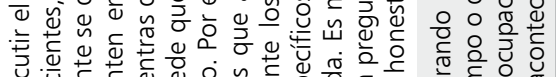

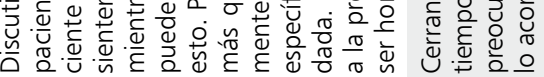


Al comparar ambos protocolos de entrega de malas noticias, es posible visualizar la importancia de incluir a la familia/otros significativos dentro de este proceso, buscando que tanto ellos, pero por sobretodo el paciente, comprenda la información entregada por el tratante, siendo este objetivo transversal a lo largo del proceso de entrega de malas noticias. Las diferencias entre ambos son mínimas, quizás cabría mencionar que el protocolo de Bennett \& Alison se inicia con un proceso de preparación previa a la entrega de la mala noticia.

\section{Judicialización de la medicina}

Este factor ha tomado preponderancia este último tiempo, debido al aumento de demandas por negligencias médicas, por lo que la adecuada entrega de información al paciente toma un protagonismo mayor.

En este sentido, el derecho a la información es un Derecho Humano consagrado en diversos Tratados Internacionales ratificados por Chile. La Convención Americana de Derechos Humanos, la Declaración Universal de Derechos Humanos y el Pacto Internacional de Derechos Civiles y Políticos, establecen un derecho positivo a buscar y a recibir información por lo que se puede deducir que existe una obligación legal y moral de informar al paciente sobre su diagnóstico, independiente de cual sea el pronóstico (Cornejo MI. Derechos y deberes de las personas en los sistemas de salud: análisis bioético de la Ley No 20.584 (Tesis para optar al grado de Magíster en Derecho). Universidad de Chile, Chile. 2014).

En la literatura está descrito que la decisión del médico de revelar o no el diagnóstico de cáncer está determinada por varios factores tales como la edad, inteligencia, estabilidad emocional, personalidad, apoyo psicológico de amigos y familiares, y el deseo de amigos y familiares de proteger al paciente con cáncer del diagnóstico ${ }^{11,12,59}$. Sin embargo, los procesos de judicialización existente dan a entender que la entrega de información al paciente debe considerarse como un derecho, más que una decisión propia del médico.

Diversas investigaciones en países occidentales muestran que los pacientes quieren saber sobre su diagnóstico de cáncer, sin embargo, en otros países como Arabia Saudita, Japón, Europa del Este, Grecia, Italia y Polonia, los pacientes con cáncer no realizan preguntas acerca del cáncer o simplemente no son informados de su diagnóstico ${ }^{59}$. Esto se debe a importantes diferencias culturales ya que los países asiáticos, árabes y africanos presentan una orientación hacia la familia, en donde ésta decide si el paciente debe ser informado o no, versus los países anglosajones y de Europa del Norte que poseen una orientación hacia el individuo y su autonomía ${ }^{59}$.

En relación a la preferencia de pacientes sobre la información que desean recibir, un estudio realizado en la Unidad de Cuidados Paliativos del Hospital Sótero del Río reveló que la mayoría de los pacientes (89-90\%) prefiere ser informado completamente sobre su diagnóstico y pronósti$\mathrm{Co}^{60}$. Lo anterior es concordante con lo reportado por diversas investigaciones, en donde, entre 79\% a $98 \%$ de los pacientes desea saber su diagnóstico ${ }^{61}$.

\section{Conclusión}

Comunicar una mala noticia al paciente de una manera adecuada no es una habilidad opcional, es más bien es una parte esencial de la práctica profesional $^{4}$, motivo por el cual este tópico cada vez cobra mayor relevancia.

El debate acerca del nivel de veracidad de la información dada a los pacientes sobre su diagnóstico se ha desarrollado significativamente este último tiempo. Mientras que los médicos y profesionales de la salud han ido incrementando la información compartida, la práctica hasta entonces ha sido retener información debido a la creencia que existe de que el profesional de la salud sabe (y decide) lo que es mejor para los intereses del paciente.

Existen antecedentes de que los médicos no les informan a los pacientes el diagnóstico de cáncer, particularmente en pacientes de mayor edad ${ }^{52-53}$. Esto a pesar de la evidencia de que algunos pacientes con tumores desean saber si su enfermedad es cáncer, y otros quieren saber lo más posible acerca de su enfermedad. A menudo más de un médico asume lo que los pacientes quieren saber ${ }^{54-56}$.

Al mismo tiempo, ha sido una práctica común en algunas áreas de las disciplinas biomédicas el entregar gran cantidad de información confidencial a los familiares sin el consentimiento expreso del paciente, incluso antes de que el paciente sepa de su condición. Aunque cada caso es diferente, 
se debe ser cuidadoso y considerar plenamente las necesidades del paciente y su familia cuando se está entregando la información ${ }^{4}$.

La evidencia indica que los pacientes han mostrado mayor interés en conocer información adicional de su diagnóstico, de sus cambios en la cura, los efectos adversos de la terapia y una estimación realista de cuánto tiempo van a vivir ${ }^{54}$. Los pacientes quieren que su médico sea honesto, compasivo, cuidadoso, esperanzador e informativo. Quieren que se les entregue la información personalmente, en un lugar privado, a su ritmo, con tiempo para la discusión, y si ellos desean, con una persona de apoyo presente ${ }^{56}$. Además, la entrega del diagnóstico y pronóstico de una enfermedad es una decisión que debe ser tomada en conjunto, tanto entre el médico tratante-familia, y debe ser una decisión informada, la cual debe ser a su vez, registrada en la ficha clínica de cada paciente.

Dentro de las proyecciones, resulta fundamental realizar una evaluación y catastro del cómo se están entregando las malas noticias en Chile, ya que el número de estudios son bastante limitados. Además sería importante profundizar en el tipo de información entregada, si se involucra a la familia/ otro significativo, entre otras cosas.

\section{Referencias}

1. Sweeny K, Shepperd JA, Han P K. The goals of communicating bad news in health care: do physicians and patients agree? Health Expec 2011; 16 (3): 230-8.

2. Finlay I, Dallimore D. Your child is dead. Bmj 1991; 302 (6971): 1524-5.

3. Baile W, Buckman R, Beale E, Lenzi R, Glober G, Beale EA, et al. Spikes-A Six-Step Protocol for Delivering Bad News: Application to the Patient with Cancer. Oncologist 2000; 5 (4): 302-11.

4. Department of health, social service \& public safety (DHSSPS). (2003). Breaking bad news, Regional guidelines. Recuperado de http://www.dhsspsni.gov.uk/ breaking_bad_news.pdf.

5. Back AL, Arnold RM, Quill TE. Hope for the best, and prepare for the worst. Ann Intern Med 2003; 138 (5): 439-43.

6. Dias L, Chabner BA, Lynch TJ Jr, Penson RT. Breaking bad news: a patient's perspective. Oncologist 2003; 8 (6): 587-96.

7. Bor R, Miller R, Goldman E, Scher I. The Meaning of Bad News in HIV Disease: counselling about dreaded issues revisited. Counsel Psychol 1993; 6: 69-80.
8. Bennett $\mathrm{M}$, Alison D. Discussing the diagnosis and prognosis with cancer patients. Postgrad Med J 1996; 72 (843): 25-9.

9. Buckman R. How to break bad news: a guide for healthcare professionals. Baltimore: Johns Hopkins University Press; 1992.

10. Silverman J, Kurtz S, Draper J. Skills for communicating with patients. Oxford: Radcliffe Publishing; 2005.

11. Fallowfield L. Giving Sad and Bad News. Lancet 1993; 341 (8843): 477-8.

12. Quirt CF, Mc Killop WJ, Ginsburg AD, Heldon L, Brundage $M$, Dixon $P$, et al. Do doctors know when their patients don't? A survey of doctor-patient communication in lung cancer. Lung Cancer 1997; 18 (1): 1-20.

13. Weeks JC, Cook EF, O’ Day SJ, Peterson LM, Wenger $\mathrm{N}$, Reding $\mathrm{D}$, et al. Relationship between cancer patients' predictions of prognosis and their treatment preferences. JAMA 1998: 279 (21); 1709-14.

14. Haidet P, Hamel MB, Davis RB, Wenger N, Reding D, Kussin PS, et al. Outcomes, preferences for resuscitation, and physician-patient communication among patients with metastatic colorectal cancer. SUPPORT investigators. Study to Understand Prognoses and Preferences for Outcomes and Risks of Treatments. Am J Med 1998; 105 (3): 222-9.

15. Ford S, Fallowfield L, Lewis S. Doctor-patient interactions in oncology. Soc Sci Med 1996; 42 (11): 1511-9.

16. Butow PN, Dunn SM, Tattersall MH. Communication with cancer patients: does it matter? J Palliat Care 1995; 11 (4): 34-8.

17. Sardell AN, Trierweiler SJ. Disclosing the cancer diagnosis. Procedures that influence patient hopefulness. Cancer 1993; 72 (11): 3355-65.

18. Roberts CS, Cox CE, Reintgen DS, Baile WF, Gibertini M. Influence of physician communication on newly diagnosed breast cancer patients' psychologic adjustment and decision-making. Cancer 1994; 74 (1 suppl): 336-41.

19. Last BF, van Veldhuizen AM. Information about diagnosis and prognosis related to anxiety and depress in children with cancer aged 8-16 years. Eur J Cancer 1996; 32A (2): 290-4.

20. Fallowfield LJ, Hall A, Maguire GP, Baum M. Psychological outcomes of different treatment policies in women with early breast cancer outside a clinical trial. Br Med J 1990; 301 (6752): 575-80.

21. Hawighorst S, Schoenefuss G, Fusshoeller C, Franz C, Seufert R, Kelleher DK, et al. The physician-patient relationship before cancer treatment: a prospective longitudinal study. Gynecol Oncol 2004; 94 (1): 93-7. 
22. Fallowfield L, Jenkins V. Effective communication skills are the key to good cancer care. Eur J Cancer 1999; 35 (11): 1592-7.

23. Ellis PM, Tattersall MH. How should doctors communicate the diagnosis of cancer to patients? Ann Med 1999; 31 (5): 336-41.

24. Liénard A, Merckaert I, Libert Y, Delvaux N, Marchal S, Boniver J, et al. Factors that influence cancer patients' anxiety following a medical consultation: impact of a communication skills training programme for physicians. Ann oncol 2006; 17 (9): 1450-8.

25. House A, Stark D. Anxiety in medical patients. Br Med J 2002; 325 (7357): 207-9.

26. van der Molen B. Relating information needs to the cancer experience: 1 . Information as a key coping strategy. Eur J Cancer Care 1999; 8 (4): 238-44.

27. Razavi D, Delvaux N. Communication skills and psychological training in oncology. Eur J Cancer 1997; 33 (suppl 6): S15-S21.

28. Jones FME, Fellows JL, Horne DJ de L. Coping with cancer: a brief report on stress and coping strategies in medical students dealing with cancer patients. Psychooncology 2011; 20 (2): 219-23.

29. Taylor SE. Health Psychology, 6ta Ed. Boston: McGrawHill; 2005.

30. Whitney SN, McCullough LB, Frugé E, McGuire Al, Volk RJ. Beyond breaking bad news, the role of hope and hopefulness. Cancer 2008; 113 (2): 442-5.

31. Back AL, Curtis AR. Communicating bad news. West J Med 2002; 176 (3): 177-80.

32. Freedman B. O ering truth: one ethical approach to the uniformed cancer patient. Arch Intern Med 1993; 153 (5): 572-6.

33. Fallowfield L, Jenkins V, Beveridge HA. Truth may hurt but deceit hurts more: communication in palliative care. Palliat Med 2002; 16 (4): 297-303.

34. Salander P. Bad news from the patients perspective: an analysis of the written narratives of newly diagnosed cancer patients. Soc Sci Med 2002; 55 (5): 721-32.

35. Clayton JM, Butow PN, Arnold RM, Tattersall MHN. Fostering coping and nurturing hope when discussing the future with terminally ill cancer patients and their caregivers. Cancer 2005; 103 (9): 1965-75.

36. Lerman C, Daly M, Walsh WP, et al. Communication between patients with breast cancer and health care providers: determinants and implications. Cancer 1993; 72 (9): 2612-20.

37. Butow PN, Kazemi JN, Beeney LJ, Gri n A, Dunn SM, Tattersall MH. When the diagnosis is cancer: patient communication experiences and preferences. Cancer 1996; 77 (12): 2630-7.
38. Ellis PM, Tattersall MH. How should doctors communicate the diagnosis of cancer to patients? Ann Med 1999; 31 (5): 336-41.

39. Dunn SM, Butow PN, Tattersall MH, Jones QJ, Sheldon JS, Taylor JJ, et al. General information tapes inhibit recall of the cancer consultation. J Clin Oncol 1993; 11 (11): 2279-85

40. Yates P. Toward a reconceptualization of hope for patients with a diagnosis of cancer. J Adv Nurs 1993; 18 (5): 701-6.

41. Groopman J. The Anatomy of Hope: How People Prevail in the Face of Illness. New York: Random House, 2003.

42. Ambuel B, Mazzone MF. Breaking bad news and discussing death. Prim Care 2001; 28 (2): 249-67.

43. Ramírez AJ, Graham J, Richards MA, Cull A, Gregory WM, Leaning MS, et al. Burnout and psychiatric disorder among cancer clinicians. Br J Cancer 1995; 71 (6): 1263-9.

44. Baile WF, Aaron J. Patient-physician communication in oncology: past, present, and future. Curr Opin Oncol 2005; 17 (4): 331-5.

45. Fallowfield L, Jenkins V. Communicating sad, bad, and difficult news in medicine. Lancet 2004; 363 (9405): 3129.

46. Ptacek JT, Eberhardt TL. Breaking bad news: a review of the literature. JAMA 1996; 276 (6): 496-502.

47. Shields CE. Giving patients bad news. Prim Care 1998; 25 (2): 381-90.

48. Baile WF, Kudelka AP, Beale EA, Glober GA, Myers EG, Greisinger AJ, et al. Communication skills training in oncology-description and preliminary outcomes of workshop on breaking bad news and managing patient reactions to illness. Cancer 1999; 86 (5): 887-97.

49. Back AL, Arnold RM, Baile WF, Tulsky JA, FryerEdwards K. Approaching difficult communication tasks in oncology. CA Cancer J Clin 2005; 55 (3): 164-77.

50. Finlay E, Casarett D. Making difficult discussions easier: Using prognosis to facilitate transitions to hospice. CA Cancer J Clin 2009; 59 (4): 250-63.

51. Kaplan M. Spikes: a framework for breaking bad news to patients to patients with cancer. Clin J Oncol Nurs 2010; 14 (4): 514-6.

52. Seifart C, Hofmann M, Bär T, Riera Knorrenschild J, Seifart U, Rief W. Breaking bad news-what patients want and what they get: evaluating the SPIKES protocol in Germany. Ann Oncol 2014; 25 (3): 707-11.

53. Bonnaud-Antignac A, Campion L, Pottier P, Supiot S. Videotaped simulated interviews to improve medical students' skills in disclosing a diagnosis of cancer. Psychooncology 2010; 19 (9): 975-81.

54. Wuensch A, Tang L, Goelz T, Zhang Y, Stubenrauch S, 
Song L, et al. Breaking bad news in China-the dilemma of patients' autonomy and traditional norms. A first communication skills training for Chinese oncologists and caretakers. Psychooncology 2013; 22: 1192-5.

55. Seeman MV. Breaking bad news: schizophrenia. J Psychiatr Pract 2010; 16 (4): 269-76.

56. Spafford MM, Schryer CF, Creutz S. Balancing patient care and student education: learning to deliver bad news in an optometry teaching clinic. Adv Health Sci Educ Theory Pract 2009; 14 (2): 233-50.

57. Shaw JR, Lagoni L. End-of-life communication in veterinary medicine: delivering bad news and euthanasia decision making. Vet Clin North Am Small Anim Pract 2007; 37 (1): 95-108.

58. Güneri P, Epstein J, Botto RW. Breaking bad medical news in a dental care setting. J Am Dent Assoc 2013; 144
(4): 381-6.

59. Wang SY, Chen CH, Chen YS, Huang HL. The attitude toward truth telling of cancer in Taiwan. J Psychosom Res 57 (2004) 53-8.

60. Palma A, Cartes F, González M, Villarroel L, Afonseca Parsons H, Yennurajalingam S, Bruera E. ¿Cuánta información desean recibir y cómo prefieren tomar sus decisiones pacientes con cáncer avanzado atendidos en una Unidad del Programa Nacional de Dolor y Cuidados Paliativos en Chile? Rev Med Chile 2014; 142: 48-54.

61. Motlagh A, Yaraei N, Mafi AR, Hosseini K, Yaseri M, Hemati S, et al. Attitude of Cancer Patients toward Diagnosis Disclosure and their Preference for Clinical Decision-making: A National Survey. Arch Iran Med 2014; 17 (4): 232-40. 